\title{
Differentiation of tin oxides using electron energy-loss spectroscopy
}

\author{
M. S. Moreno* \\ Centro Atómico Bariloche, 8400 San Carlos de Bariloche, Argentina \\ R. F. Egerton \\ Department of Physics, University of Alberta, Edmonton, Canada T6G $2 \mathrm{J1}$ \\ P. A. Midgley \\ Department of Materials Science and Metallurgy, University of Cambridge CB2 3QZ, United Kingdom
}

(Received 19 December 2003; published 9 June 2004)

\begin{abstract}
We have used electron energy-loss spectroscopy to search for differences in the energy-loss near-edge structure of $\mathrm{SnO}, \mathrm{SnO}_{2}$, and an intermediate oxide, with a view to distinguishing them unambigously. We have found that the oxygen $K$ edge exhibits clear differences that can be used for fingerprinting each phase. The oxygen edge appears at the same position for each phase whereas a chemical shift of the $\mathrm{Sn} M_{4,5}$ edge of about $3.5 \mathrm{eV}$ was observed between phases with $\mathrm{Sn}$ in $2+$ and $4+$ oxidation states. Both observations can be used to distinguish between the three phases, allowing their on-line identification within nanostructured materials.
\end{abstract}

DOI: 10.1103/PhysRevB.69.233304

PACS number(s): 79.20.Uv, 82.80.Pv, 61.10.Ht

Tin and its oxides are technologically important materials with several applications, for example, in heterogeneous catalysis and as semiconductor and gas sensing devices, ${ }^{1}$ solar energy cells, and lithium ion batteries., ${ }^{2,3}$ These uses can require bulk or nanostructured materials ${ }^{4,5}$ or thin films. ${ }^{6,7}$

Many synthesis routes involve change in the oxidation state of tin, from $\mathrm{Sn}(0)$ to $\mathrm{Sn}$ (II) or $\mathrm{Sn}$ (II) to $\mathrm{Sn}(\mathrm{IV})$, or else the presence of more than one phase, often hard to characterize by standard techniques because of the small domain size. Another difficulty for processes that involve change in the oxidation state from $2+$ to $4+$ is that $\mathrm{SnO}$ undergoes thermal decomposition involving a metastable phase, usually called the intermediate oxide (IO), which requires high temperatures to be completely converted to $\mathrm{SnO}_{2}{ }^{8}$ Although high energy electron diffraction has the sensitivity and spatial resolution to identify these phases, the difference in reciprocal lattice between the stable oxides $[a=3.8029 \AA, c$ $=4.8382 \AA$ for SnO (Ref. 9) and $a=4.7373 \AA, c=3.1864 \AA$ for $\mathrm{SnO}_{2}$ (Ref. 10)] is small (except for certain zone axes) and difficult to resolve by diffraction techniques. In practice this means that tilting experiments are needed to make sure of the identity of the phase(s) under study, a requirement which can be inconvenient and tedious in the case of finescale (nanostructured) materials. This suggests the need for a practical, fast, and unambiguous method for distinguishing between the different oxides $\left(\mathrm{SnO}_{2}, \mathrm{SnO}\right.$, and IO) at high spatial resolution, for example, by acquiring information on the elemental composition and chemical state.

For this purpose, many low-energy surface techniques (such as Auger spectroscopy) fail. ${ }^{11}$ The capability of x-ray photoelectron spectroscopy (XPS) to distinguish tin in its oxidized forms ( $\mathrm{SnO}$ and $\mathrm{SnO}_{2}$ ) has been discussed at length. It is accepted that a sizable chemical shift (about $0.7 \mathrm{eV}$ ) exists between formal $\mathrm{Sn}^{4+}$ and $\mathrm{Sn}^{2+}$, as detected by XPS. ${ }^{12}$ But XPS is highly surface sensitive and requires the use of sputter cleaning to remove the thick layer of $\mathrm{SnO}_{2}$ which is formed on air oxidized $\mathrm{SnO}$. It has recently been shown, from changes in a high-resolution electron microscopy image or electron-diffraction pattern, ${ }^{13}$ that electron-beam damage is observable in $\mathrm{SnO}$ under standard working conditions. The extent of the damage after sputtering with low-energy argon ions is therefore unknown. A problem related to surface effects induced by sputtering in $\mathrm{SnO}_{2}$ is the reduction of half of the surface Sn atoms from $\mathrm{Sn}(\mathrm{IV})$ to $\mathrm{Sn}(\mathrm{II})$ after removal of the bridging oxygen ions that are normally present in the stoichiometric surface. ${ }^{14}$ Although the IO is always present in samples that involves the transformation of $\mathrm{SnO}$ to $\mathrm{SnO}_{2}$, it has not been considered in previous studies. Its composition is uncertain, although several possibilities have been proposed, the more probable ones being $\mathrm{Sn}_{2} \mathrm{O}_{3}$ and $\mathrm{Sn}_{3} \mathrm{O}_{4}$. However our characterization of this compound by electron microscopy techniques suggest that the compound is single phase. ${ }^{15}$ Despite the ambiguity in its composition, we have included the IO phase in our present study because it includes both oxidation states of tin and might allow a more complete distinction between the different tin oxides.

Electron microscopy provides a spatial resolution adequate to distinguish each phase individually, even in multiphasic samples. In addition to its bulk sensitivity, electron energy-loss spectroscopy (EELS), unlike XPS, involves transitions between two states: the core level and an unoccupied state, allowing the possibility of a different valencedependent chemical shift. An added advantage is that (in many cases) the observed EEL spectrum exhibits a structure that is specific to the nearest-neighbor coordination, and can be used as a coordination fingerprint. ${ }^{16}$ The oxygen atoms are in different coordination polyhedra in $\mathrm{SnO}$ and $\mathrm{SnO}_{2}$. In the latter case, oxygen is in a triangular coordination while in the former case it is surrounded by a regular tetrahedron of cations. Here we explore the capability of transmission EELS carried out in a transmission electron microscope (TEM) to detect robust differences in the $\mathrm{Sn}$ and $\mathrm{O}$ ionization edges of the different oxide phases, in order to provide an empirical but nonsubjective method of identification.

We used commercial (Aldrich) powders of $\mathrm{SnO}$ and $\mathrm{SnO}_{2}$, in addition to the intermediate compound formed by 


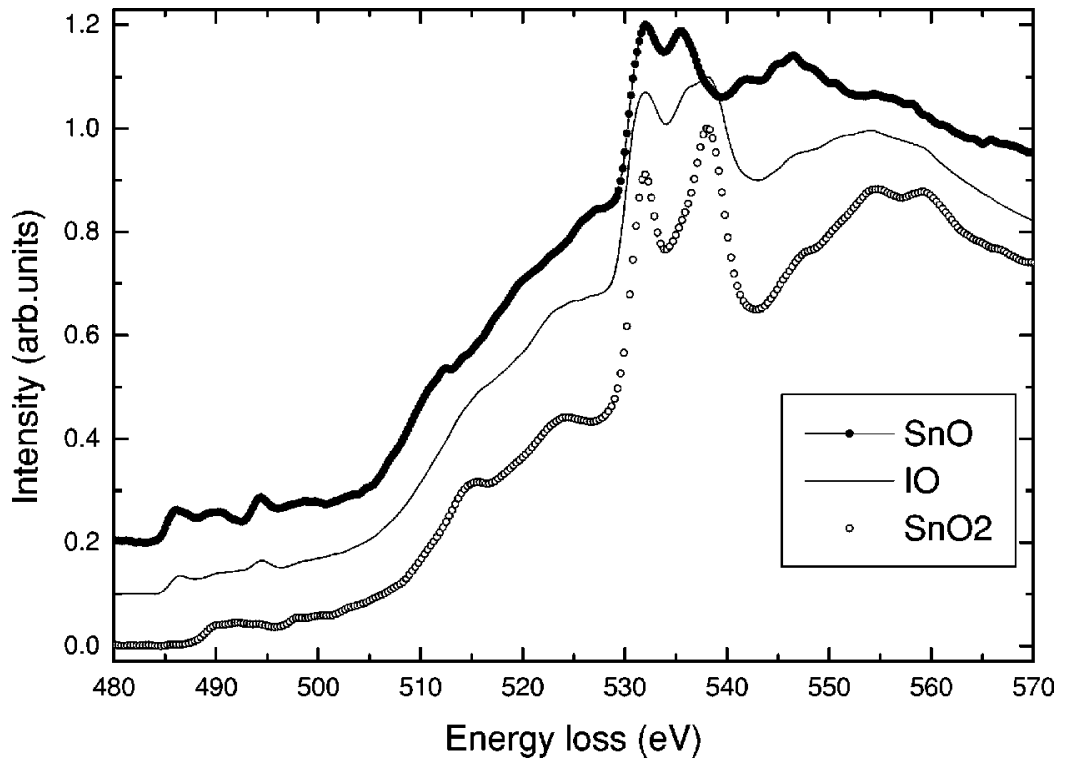

FIG. 1. EELS spectra of $\mathrm{SnO}, \mathrm{SnO}_{2}$, and the intermediate oxide. The spectra were vertically shifted for comparison.

thermal decomposition of $\mathrm{SnO}$ at $723 \mathrm{~K}$ in a vacuum of $10^{-3}$ Torr for $1 \mathrm{~h} .{ }^{17}$ The characteristic $\sim 0.8 \mathrm{~nm}$ lattice spacing of this intermediate phase enabled it to be distinguished among the multiphasic products of $\mathrm{SnO}$ thermal decomposition. A detailed characterization of this phase will be published separately. ${ }^{15}$

Energy-loss spectra were measured in diffraction mode with a collection semiangle of 6 mrad. A Tecnai $F 20\left(G^{2}\right)$ TEM was used coupled to a GIF 2002 spectrometer and operated at $200 \mathrm{kV}$, with the specimen at room temperature. The energy resolution, estimated from the full width at half maximum of the zero loss peak, was $1.2 \mathrm{eV}$. Because $\mathrm{SnO}$ is a beam sensitive material, we verified that our specimen remained undamaged (at the dose used for the experiments) by monitoring the electron-diffraction pattern and the energyloss spectrum for possible structural and/or electronic changes.

Typical spectra are shown in Fig. 1, where it can be seen that the $\mathrm{O}-K$ edge follows and substantially overlaps with the $M_{4}$ and $M_{5}$ delayed edges of tin. Despite this difficulty, it is possible to distinguish clear differences in the energy-loss near-edge structure (ELNES) that can be used as a fingerprint of each phase. In fact, the overlapping of both edges allows us to inspect for possible energy miscalibrations.

Full multiple scattering calculations using the FEFF 8.20 program have shown ${ }^{18}$ that the pronounced fine structure observed above $530 \mathrm{eV}$ is entirely due to oxygen states and that tin makes the main contribution below this energy. It can be observed that the separation between the $M_{5}$ edge of tin (at 486 for $\mathrm{SnO}$ and the $\mathrm{IO}$, and $490 \mathrm{eV}$ for $\mathrm{SnO}_{2}$ ) and the first oxygen peak (at $532 \mathrm{eV}$ ) is characteristic of each phase. This feature introduces the need for a better estimate of the energy scale, in order to remove the ambiguity about how to align them.

For this purpose we performed an additional experiment to check the relative position of the EELS spectra between $\mathrm{SnO}_{2}$ and the IO. This experiment consisted of preparing a sample containing both phases and recording the core-loss spectra from each phase. The results showed that the abso- lute position of the spectral features is affected by the ripple and drift of the high tension. The first factor is largely averaged when using a long acquisition time.

We plot in Fig. 2 the time dependence (for both phases) of the position of the $M_{5}$ edge [2(a) or upper panel] and of the first oxygen peak [2(b) or middle panel], respectively. The

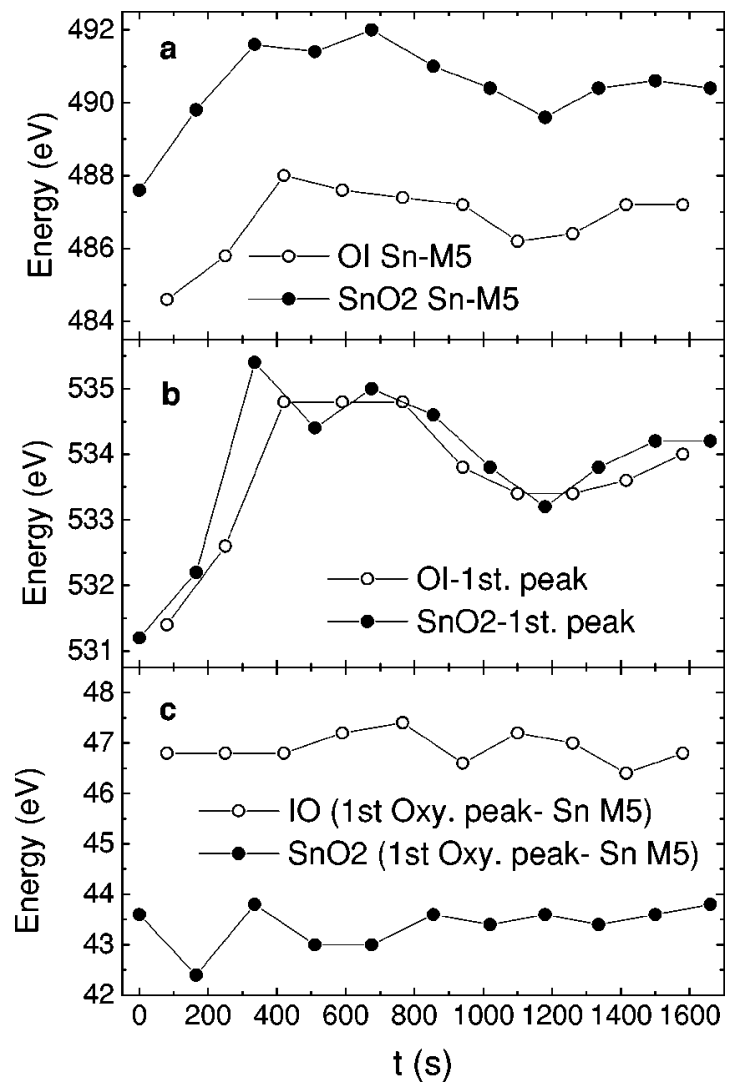

FIG. 2. Evolution with time (see the text) of the position of the $\mathrm{Sn}-\mathrm{M}_{5}$ for $\mathrm{SnO}_{2}$ and the IO [upper panel, (a)], and position of the first peak of the $\mathrm{O}-\mathrm{K}$ edges of $\mathrm{SnO}_{2}$ and the intermediate oxide [medium panel, (b)]. In the lower panel is plotted the difference between the first peak of oxygen and the Sn $M_{5}$ edge. 

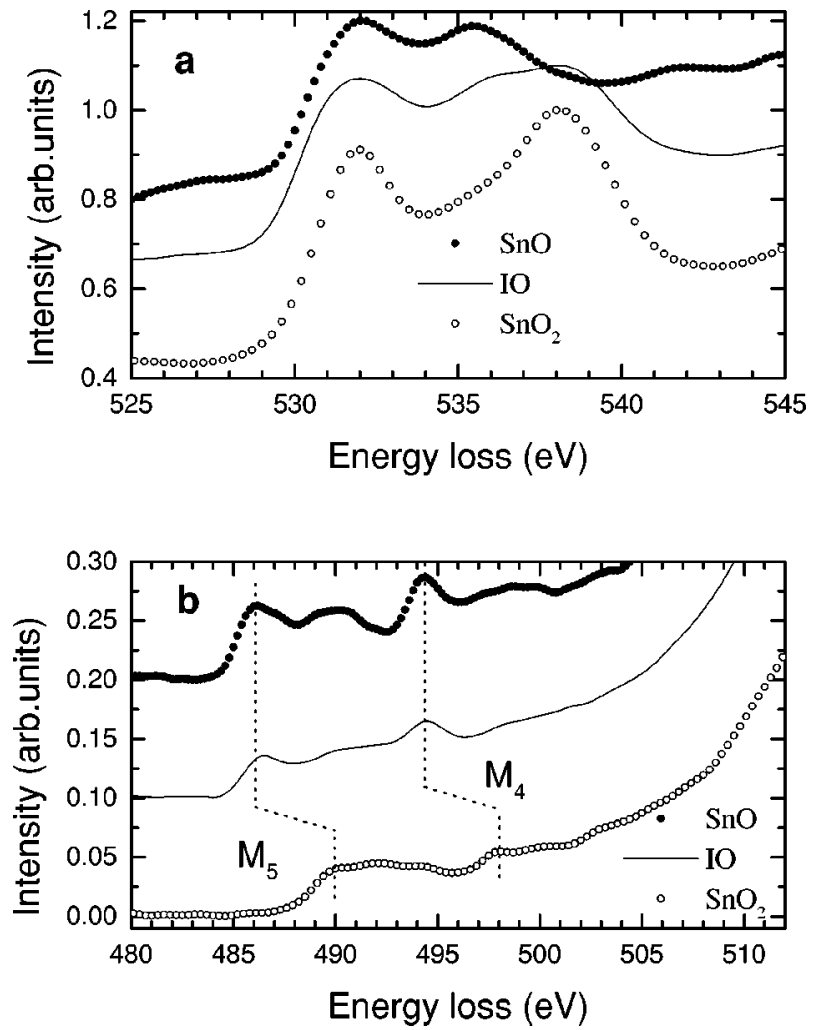

FIG. 3. Expanded region of Fig. 1, corresponding to (a) the O- $K$ edge and (b) the $\mathrm{Sn}-\mathrm{M}_{4,5}$ edges. Line dots are a guide to the eye.

observed changes are due to drift of the high tension, and possibly of the spectrometer power supply. The nearly parallel evolution in Fig. 2(a), without intercrossing, strongly suggests that the position of the $M_{5}$ edge is different in each phase. In the same way, the close position of both curves in Fig. 2(b) suggests that the first peak of the oxygen fine structure remains at roughly the same position. This evidence therefore suggests that it is reasonable to align the spectra by taking the first oxygen peak as a reference, leaving absolute energies undetermined. In this way, we avoid the need for one of the few instruments in the world in which the energyscale instabilities are corrected or minimized.

In Fig. 1, we have aligned the spectra with respect to the first oxygen peak (set arbitrarily at $532 \mathrm{eV}$ ) in accord with the above principle. Our spectrum for $\mathrm{SnO}_{2}$ is then in agreement with that previously reported for the oxygen edge by x-ray absorption spectroscopy. ${ }^{19}$ It can be seen that the oxygen fine structure of $\mathrm{SnO}$ and $\mathrm{SnO}_{2}$ is entirely different in both phases, particularly in the energy range $530-540 \mathrm{eV}$ [Fig. 3]. In this region, $\mathrm{SnO}_{2}$ shows a fine structure consisting of three (or possibly four) peaks with separations of at least $6 \mathrm{eV}$ whereas $\mathrm{SnO}$ in the same region displays two peaks spread over a narrower energy interval of approximately $3.5 \mathrm{eV}$. The oxygen fine structure of the IO seems to be a combination of both cases.

Other differences appear in the region $540-570 \mathrm{eV}$ in the form of a different number of peaks and in the energy of them that can be also used to distinguish between the two compounds. Since these features seem to be multiple scattering resonances which should go as $1 /$ (bond length) ${ }^{2}$ we at- tribute these differences to the dissimilar crystal structures, with quite distinct $\mathrm{Sn}-\mathrm{O}$ nearest-neighbor distances: $0.206 \mathrm{~nm}$ and $0.222 \mathrm{~nm}$ for $\mathrm{SnO}_{2}$ and $\mathrm{SnO}$, respectively.

The features in the first few $\mathrm{eV}$ of the $\mathrm{O} K$-ELNES are dominated by transitions to $\mathrm{O} 2 p$ or $\mathrm{O} 2 p$-Sn $5 p$ hybridized empty states. The crystal structures of $\mathrm{SnO}$ and $\mathrm{SnO}_{2}$ possess only one nonequivalent site for both atoms, then the observed O $K$-ELNES reflects the different arrangements, tetrahedral and triangular respectively, in the first coordination shell of oxygen and can be clearly used for fingerprinting each phase. In the case of the IO its crystal structure is unknown and the features observed would be of help in its identification. In fact, we have noticed that its ELNES seems to be a combination of those of $\mathrm{SnO}$ and $\mathrm{SnO}_{2}$ suggesting the existence of more than one crystallographic (and chemical) site for oxygen, as might be expected for a compound with both oxidation states.

In Fig. 3(b) the region of the $M_{5}$ and $M_{4}$ edges of tin is shown expanded. It can be observed that the $M_{5}$ threshold is coincident (within experimental error) for the phases containing $\mathrm{Sn}$ in 2+ oxidation state $(\mathrm{SnO}$ and the $\mathrm{IO})$ and that a chemical shift of approximately $3.5 \mathrm{eV}$ exists for $\mathrm{SnO}_{2}$, as shown previously in Fig. 2(a). This shift is clearer in Fig. 2(c), where we plot the difference between the position of the first peak of oxygen and the $M_{5}$ edge. Therefore the $M_{5}$ energy (relative to the first oxygen peak) can be used to distinguish between phases containing $2+$ and only $4+$ oxidation states.

EELS chemical shifts are complicated phenomena ${ }^{20}$ involving two qualitatively different orbitals, the core level and the lowest energy occupied level. This makes them sensitive to the sample band structure and a full explanation would involve full electronic structure calculations which are beyond the scope of this work. Because of that we have focussed our work on an empirical use of the differences observed.

We also note in Fig. 1 the same separation of approximately $8.2 \mathrm{eV}$ between the $M_{5}$ and $M_{4}$ edges for the three phases. In comparison with $\mathrm{SnO}_{2}$, the peaks at 515 and $524 \mathrm{eV}$ (due to $M_{5}$ and $M_{4}$ edges) are more rounded in the IO phase and almost absent in $\mathrm{SnO}$.

We believe that an empirical use of the fine structure observed above $530 \mathrm{eV}$, combined with the difference between the $M_{5}$ edge and the first oxygen peak, can be used to distinguish the three phases along the lines outlined above. This is a robust criterion because we have based our differentiation strategy on the energy of the peaks and not their intensities. Our conclusion is therefore unaffected by any orientation dependence of the oxygen ELNES, by instrumental resolution or multiple scattering.

In summary, we have found that the observed ELNES above $530 \mathrm{eV}$ is almost entirely due to oxygen. The three oxides of tin show clear differences and can therefore be distinguished by electron energy-loss spectroscopy.

M.S.M. acknowledges the financial support of Fundación Antorchas through the grant (Grant No. 14022-100). Partial financial support of CONICET-Argentina and the International Atomic Energy Agency (IAEA) was also acknowledged. 
*Also at CONICET. Electronic address: smoreno@cab.cnea.gov.ar

${ }^{1}$ C. Alfonso, A. Charai, A. Armigliato, and D. Narducci, Appl. Phys. Lett. 68, 1207 (1996).

${ }^{2}$ Y. Idota, T. Kubota, A. Matsufuji, Y. Maekawa, and T. Miyasaka, Science 276, 1395 (1997).

${ }^{3}$ M. H. Chen, Z. C. Huang, G. T. Wu, G. M. Zhu, J. K. You, and Z. G. Lin, Mater. Res. Bull. 38, 831 (2003).

${ }^{4}$ E. Comini, G. Faglia, G. Sberveglieri, Z. Pan, and Z. L. Wang, Appl. Phys. Lett. 81, 1869 (2002).

${ }^{5}$ Y. Chen, X. Cui, K. Zhang, D. Pan, S. Zhang, B. Wang, and J. G. Hou, Chem. Phys. Lett. 369, 16 (2003).

${ }^{6}$ J. Lawrence, P. Lubrani, and L. Li, Surf. Coat. Technol. 137, 235 (2001).

${ }^{7}$ V. M. Jimenez et al., Thin Solid Films 353, 113 (1999).

${ }^{8}$ M. S. Moreno, J. Desimoni, A. G. Bibiloni, M. Renteria, C. P. Massolo, and K. Freitag, Phys. Rev. B 43, 10086 (1991).

${ }^{9}$ J. Pannetier and G. Denes, Acta Crystallogr., Sect. B: Struct. Crystallogr. Cryst. Chem. 36, 2763 (1980).

${ }^{10}$ R. W. G. Wyckoff, Crystal Structures (Wiley, New York, 1965),
Vol. 1, p. 251.

${ }^{11}$ G. B. Hoflund and G. R. Corallo, Phys. Rev. B 46, 7110 (1992).

${ }^{12}$ J.-M. Themlin, M. Chta'ib, L. Henrard, P. Lambin, J. Darville, and J.-M. Gilles, Phys. Rev. B 46, 2460 (1992).

${ }^{13}$ M. S. Moreno, R. F. Egerton, and L. C. Otero-Díaz, Philos. Mag. Lett. 83, 591 (2003).

${ }^{14}$ J.-M. Themlin, R. Sporken, J. Darville, R. Caudano, J.-M. Gilles, and R. L. Johnson, Phys. Rev. B 42, 11914 (1990).

${ }^{15}$ M. S. Moreno and P. A. Midgley (unpublished).

${ }^{16}$ R. F. Egerton, Electron Energy-Loss Spectroscopy in the Electron Microscope (Plenum, New York, 1996).

${ }^{17}$ M. S. Moreno, R. C. Mercader, and A. G. Bibiloni, J. Phys.: Condens. Matter 4, 351 (1992).

${ }^{18}$ M. S. Moreno, R. F. Egerton, P. Rez, and P. A. Midgley (unpublished).

${ }^{19}$ M. A. Figueiredo and J. Mirão, Eur. J. Mineral. 14, 1061 (2002).

${ }^{20}$ R. Brydson, EMSA Bull. 21, 57 (1991). 